\title{
Control interno de inventarios y su relación en la rentabilidad de la empresa HECALIRO JIA SAC, Tarapoto
}

\author{
Internal control of inventories and its relationship in the profitability of the company \\ HECALIRO JIA SAC, Tarapoto
}

\begin{tabular}{|c|c|}
\hline Keily Hidalgo & Mónica Bustos \\
\hline $\begin{array}{r}\text { keilyhidaldo@upeu.edu.pe } \\
\text { ORCID: 0000-0002-2732-3278 }\end{array}$ & $\begin{array}{l}\text { monica.bustos@upeu.edu.pe } \\
\text { ORCID: 0000-0003-0608-5132 }\end{array}$ \\
\hline Universidad Peruana Unión - Perú & Universidad Peruana Unión - Perú \\
\hline
\end{tabular}

Artículo recibido en enero 2019 / Arbitrado en febrero 2019 / Publicado en mayo 2019

RESUMEN | El objetivo de esta investigación fue determinar la relación existente entre el control interno de inventarios y su relación con la rentabilidad de la empresa HECALIRO JIA SAC, Tarapoto. El enfoque de estudio fue cuantitativo de tipo aplicada, con un alcance correlacional y diseño no experimental. La muestra estuvo compuesta por 14 colaboradores que laboran en las distintas áreas de la empresa. El instrumento de recolección de datos fue un cuestionario que mide el control interno de inventarios y la rentabilidad de la empresa diseñado para empresas del rubro ferretero. Los datos recolectados fueron procesados en el software paquete estadístico IBM SPSS Stadistics versión 25. Al término del estudio se llegó a la conclusión que si existe relación significativa entre el control interno de inventarios y la rentabilidad de la empresa HECALIRO JIA SAC ya que se obtuvo un Tau $b$ de Kendall $(t=\mathbf{0} .780 * * ; \boldsymbol{p}=\mathbf{0}$. 000) el cual indica que a mayor control de inventarios mayor será la rentabilidad de la empresa.

Palabras clave:

Control; Inventarios; Rentabilidad; Empresa

ABSTRACT

Keywords:
The objective of this research was to determine the existing relationship between internal inventory control and its relationship with the profitability of the company HECALIRO JIA SAC, Tarapoto. The study approach was quantitative of the applied type, with a correlational scope and non-experimental design. The sample consisted of 14 collaborators who work in the different areas of the company. The data collection instrument was a questionnaire that measures the internal control of inventories and the profitability of the company designed for companies in the hardware industry. The data collected were processed in the statistical software package IBM SPSS Statistics version 25 . At the end of the study it was concluded that if there is a significant relationship between the internal control of inventories and the profitability of the company HECALRO JIA SAC since a Kendall's tau b $(t=\mathbf{0} .780 * * ; \boldsymbol{p}=\mathbf{0}$. 000) which indicates that the greater the control of inventories, the higher the profitability of the company.

Control; Inventories; Cost effectiveness; Company 
INTRODUCCIÓN

A nivel mundial la compra y venta de repuestos de motos han venido siendo muy importantes para satisfacer las necesidades de personas naturales y jurídicas, tanto privadas y gubernamentales. La mayoría de empresas dedicadas a estas actividades ha presentado problemas en el deficiente control de inventarios, generando pérdidas económicas a largo plazo, por lo que es necesario mantener un adecuado y estricto control sobre los mismos, en "El que se puede desarrollar el análisis financiero de la empresa, observando la parte de mercadeo y el funcionamiento global de la misma" (Alvarado, 2016, p.33). Del mismo modo el deficiente control de inventarios repercute en la rentabilidad de la empresa y en la toma de decisiones de la alta gerencia.

Para el bienestar de toda empresa, de acuerdo a Barquero (2018) se debe tener:

Conocimiento y nuevos métodos en sus diferentes áreas, con la finalidad de aportar soluciones para el bienestar de la misma. En Latinoamérica las diversas empresas han percibido que los inventarios son el corazón de todo negocio, permiten avanzar en todos sus movimientos internos y externos, reduciendo las pérdidas de los productos, además manteniendo el inventario en excelentes condiciones para ser distribuido con éxito entre los consumidores finalmente (p.67)

Según Bernales (2017) afirma que en el Perú "los problemas más frecuentes a los que se enfrentan las empresas, en cuanto al sistema de control de inventarios, radica en que no se tiene conciencia de la importancia de contar con información suficiente y útil para minimizar costos, aumentar la liquidez" (p.43) mantener un nivel de inventario óptimo y realizar una gestión empresarial eficiente.

Dentro de la mayoría de las empresas uno de los puntos más críticos dentro de sus actividades económicas es el "control de sus inventarios ya que se da tanto a nivel local, nacional y porque no internacionalmente, motivo por el cual surge los procesos de control de inventarios dentro de las organizaciones, y a partir de los procesos establecidos incrementar la rentabilidad a la empresa" (Calvay, 2017, p.34).

Los inventarios representan el punto de partida en la toma de decisiones dentro de toda organización, ya que permiten la adecuada y funcional organización de la misma. Es indispensable que la empresa establezca políticas y reglas que permitan asegurar que sus inventarios no tengan riesgos de robos o que un colaborador realice un mal manejo en el registro de ingresos y salidas.

Distintos estudios han llegado a determinar que en las áreas donde se almacena los materiales siempre se debe tener en cuenta el control interno de inventarios para evitar pérdidas o mermas, que originan inconsistencias en los Kardex, dado que de esta manera se evitarán estas deficiencias que perjudican directamente a la 
rentabilidad económica de la empresa que se verá reflejada en los registros contables.

La empresa Hecaliroja JIA SAC, tiene como actividad principal, la comercialización y distribución de repuestos de moto lineal, este establecimiento comercial está ubicado en la Av. Vía de Evitamiento $N^{\circ} 402$ Tarapoto. Luego de un análisis minucioso junto al administrador se ha determinado que la empresa presenta el problema de organizar su control en los inventarios y cómo influye de manera favorable o no en la rentabilidad, por razones de tener una gestión y administración empírica, carece de políticas y normas internas, y no existen un sistema informático para gestionar funciones que hace al personal no estar capacitado, generando pérdidas de productos, deterioro de mercaderías, que no haya rotación de inventarios, perdida de ventas, que a posteriori significa pérdida de clientes; y como consecuencia la influencia en la rentabilidad, la disminución en la rentabilidad, en la creación de valor de la empresa, y en la toma de decisiones para la empresa. En base a todo lo mencionado es importante investigar y analizar el control interno de inventarios y su relación en la rentabilidad de la empresa HECALIROJA JIA SAC, el cual permitirá contrarrestar estas irregularidades y prevenir riesgos para contribuir con sus resultados y determinar su incidencia en la mejora de la rentabilidad de la empresa.

\section{MATERIALES Y MÉTODOS}

La investigación fue de enfoque cuantitativo de tipo aplicada. Este enfoque utiliza la recolección de datos para probar hipótesis con base en la medición numérica y el análisis estadístico, con el fin establecer pautas de comportamiento y probar teorías (Hernández, Fernández, \& Baptista, 2014.p.4) y aplicada porque se encarga de resolver problemas o hipótesis de trabajo de la vida productiva de la sociedad (Hernández, Fernández, \& Baptista, 2014.p.90). El alcance fue descriptiva correlacional ya que tiene la finalidad conocer la relación o grado de asociación que exista entre las 2 variables de estudio (Hernández, Fernández, \& Baptista, 2014.p.93). El diseño de investigación fue no experimental de corte transversal, el estudio se realizó sin manipular deliberada de variables (Hernández, Fernández, \& Baptista, 2014.p.152). De corte transversal porque se recolectarán los datos en un solo momento, en un tiempo único. Su propósito es describir variables y analizar su incidencia e interrelación en un momento dado. Es como "tomar una fotografía" de algo que sucede

La población y muestra estará conformada por 14 colaboradores que laboran en las distintas áreas de la empresa, edad comprendida entre 18y 60 años. Se realizará un muestreo de tipo no probabilístico por conveniencia. La ventaja de este tipo de muestra es su utilidad para poder determinar tipos de diseños de 
estudios que no requieran de la un conjunto de preguntas respecto a uno o representatividad de la población, sino una más variables a medir con el propósito de controlada y cuidadosa selección de ciertos obtener información de la investigación. En casos específicos anticipadamente en el la presente investigación se aplicó un planteamiento del problema. (Hernández, cuestionario que mide el control interno de Fernández, \& Baptista, 2014).

El instrumento de recolección de datos inventarios y la rentabilidad de la empresa, este cuestionario fue diseñado por Cecilio empleado en esta investigación fue: el (2018) para empresas del rubro ferretero. Los cuestionario. Según Hernández, Fernández $Y$ Baptista (2014) el cuestionario es un instrumento de investigación que consiste en datos recolectados fueron procesados en el software paquete estadístico IBM SPSS Stadistics versión 25 .

\section{RESULTADOS Y DISCUSIÓN}

Tabla 1. Resultados descriptivos sociodemográficos. Información Sociodemográfica

\begin{tabular}{llll}
\hline & & Frecuencia & Porcentaje \\
\hline Género & Masculino & 9 & $64.0 \%$ \\
& Femenino & 5 & $36.0 \%$ \\
& Total & 14 & $100.0 \%$ \\
Edad & $18-30$ años & 7 & $50.0 \%$ \\
& & & \\
& $31-40$ años & 5 & $36.0 \%$ \\
& $41-50$ años & 1 & $7.0 \%$ \\
& $51-65$ años & 1 & $7.0 \%$ \\
& Total & 14 & $100.0 \%$ \\
& Administración & 3 & $21.0 \%$ \\
Área de trabajo & 3 & $21.0 \%$ \\
& Contabilidad & & $21.0 \%$ \\
& Logística & 3 & $35.0 \%$ \\
& Ventas & 5 & $100.0 \%$ \\
\hline
\end{tabular}

Fuente: Hidalgo, Bustos (2019) 
La Tabla 1 muestra la información sociodemográfica de la población. Los resultados muestran que el $64 \%$ de la población está compuesta por varones y el $36 \%$ está compuesta por mujeres. La representatividad de los varones se debe a que en las ferreterías mayormente se necesita personal varón por tratarse de un trabajo forzado y expuesto a riesgos. En cuanto a la edad el $50 \%$ de la población es relativamente joven entre 18 a 30 años. El $36 \%$ de los colaboradores están entre 31 a años y paralelamente el $7 \%$ de la población está entre 41 a $50-51$ a 65 años. Por otro lado, los resultados del área de trabajo muestran que el $36 \%$ de la población pertenecen a la fuerza de ventas, en porcentajes igualitarios el $21 \%$ pertenece al área de administración, contabilidad y logística.

Tabla 2. Análisis de confiabilidad. Estadísticas de fiabilidad Alfa de Cronbach

\begin{tabular}{lcc}
\hline Variables & Alfa de Cronbach & Ítems \\
\hline Cuestionario para medir el control interno y rentabilidad & 0,82 & 14 \\
\hline
\end{tabular}

Fuente: Hidalgo, Bustos (2019)

La Tabla 2 señala que, el instrumento cuestionario para medir el control interno de inventarios y rentabilidad de la empresa obtuvo un índice de confiabilidad de 0,82; interpretándose como buena puesto que el valor debe ser mayor a 0,70 para aceptar su confiabilidad y demostrar que el instrumento fue válido para la muestra correspondiente.

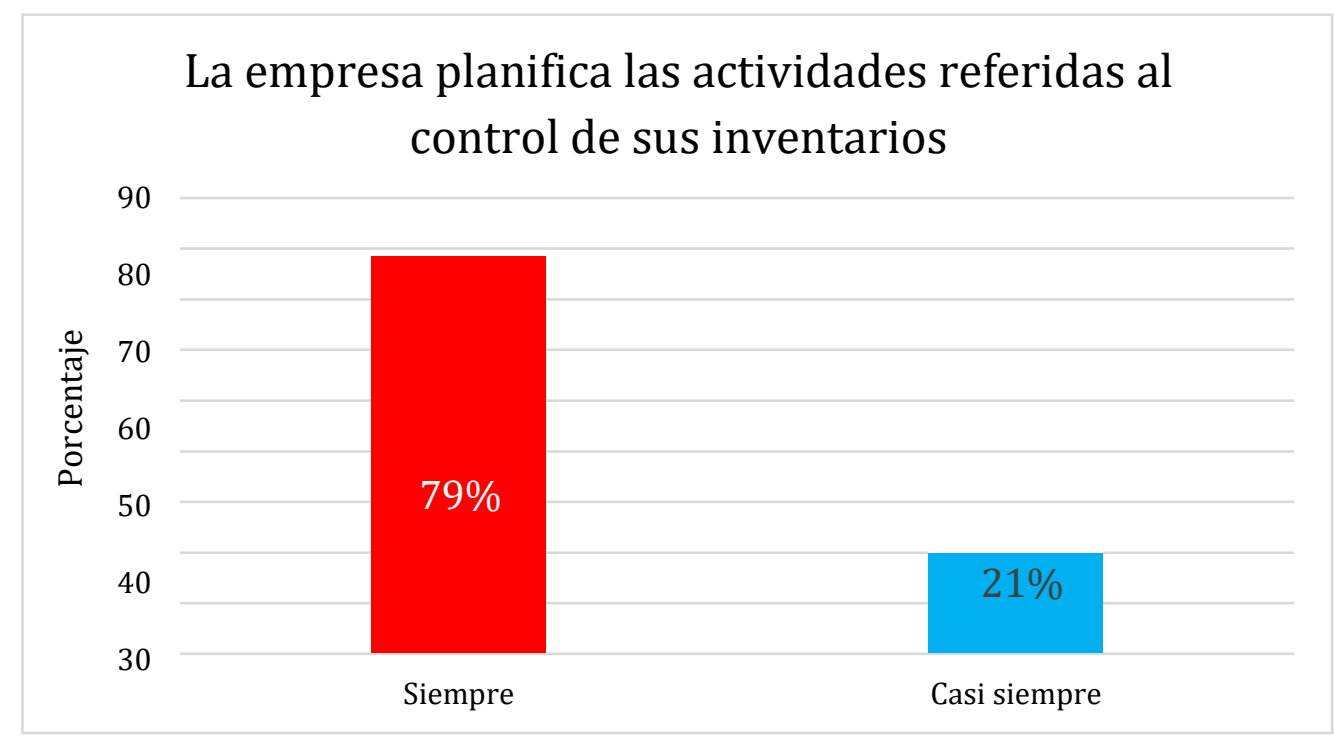

Gráfico 1. Planificación de actividades referidas al control interno 
En el gráfico 1 se presenta los resultados sobre la planificación de las actividades referidas al control de inventarios en la empresa. De acuerdo a los resultados el 79\% de los colaboradores afirman que la empresa siempre planifica las actividades de control de inventarios. Sin embargo, el $21 \%$ de la población dice que la empresa realiza esta actividad.

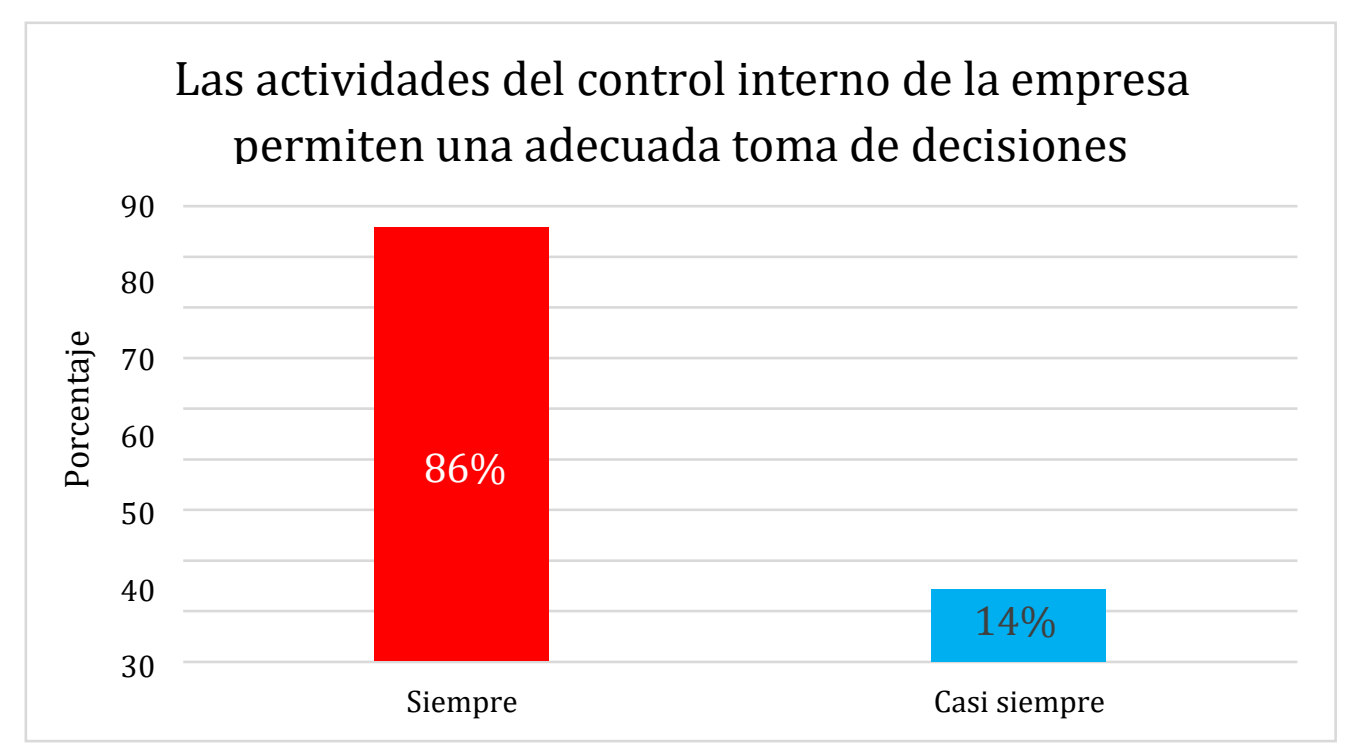

Gráfico 2. Las actividades de control interno permiten una adecuada toma de decisiones

En el gráfico 2 se aprecia los resultados si las actividades del control interno de la empresa permiten una adecuada toma de decisiones, donde el $86 \%$ de los colaboradores afirman que las actividades de control interno de la empresa siempre permiten una adecuada toma de decisiones; el $14 \%$ menciona que casi siempre permite una adecuada toma de decisiones. 


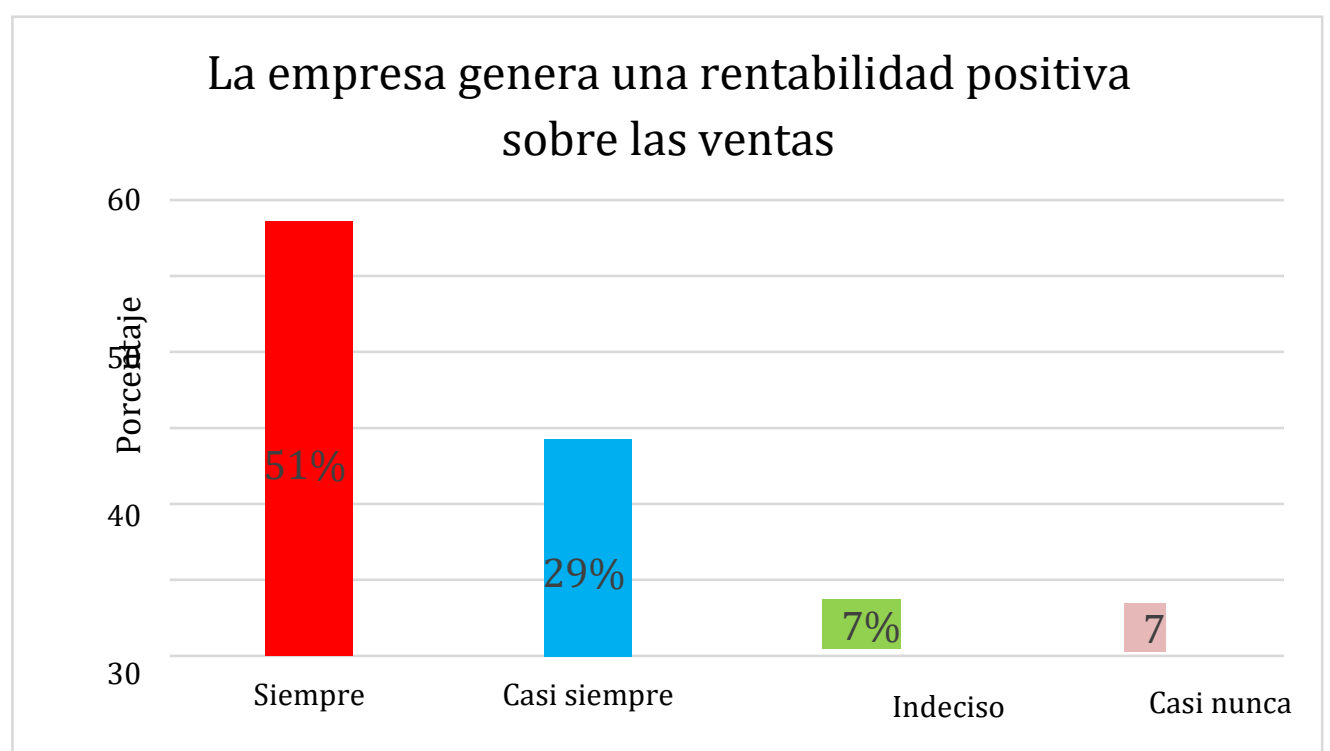

Gráfico 3. La empresa genera una rentabilidad positiva sobre las ventas

En el gráfico 3 se observa que el $51 \%$ de los colaboradores afirma que la empresa siempre genera una rentabilidad positiva sobre las ventas, el $29 \%$ de encuestados mencionan que casi siempre y un $7 \%$ se muestra indeciso.

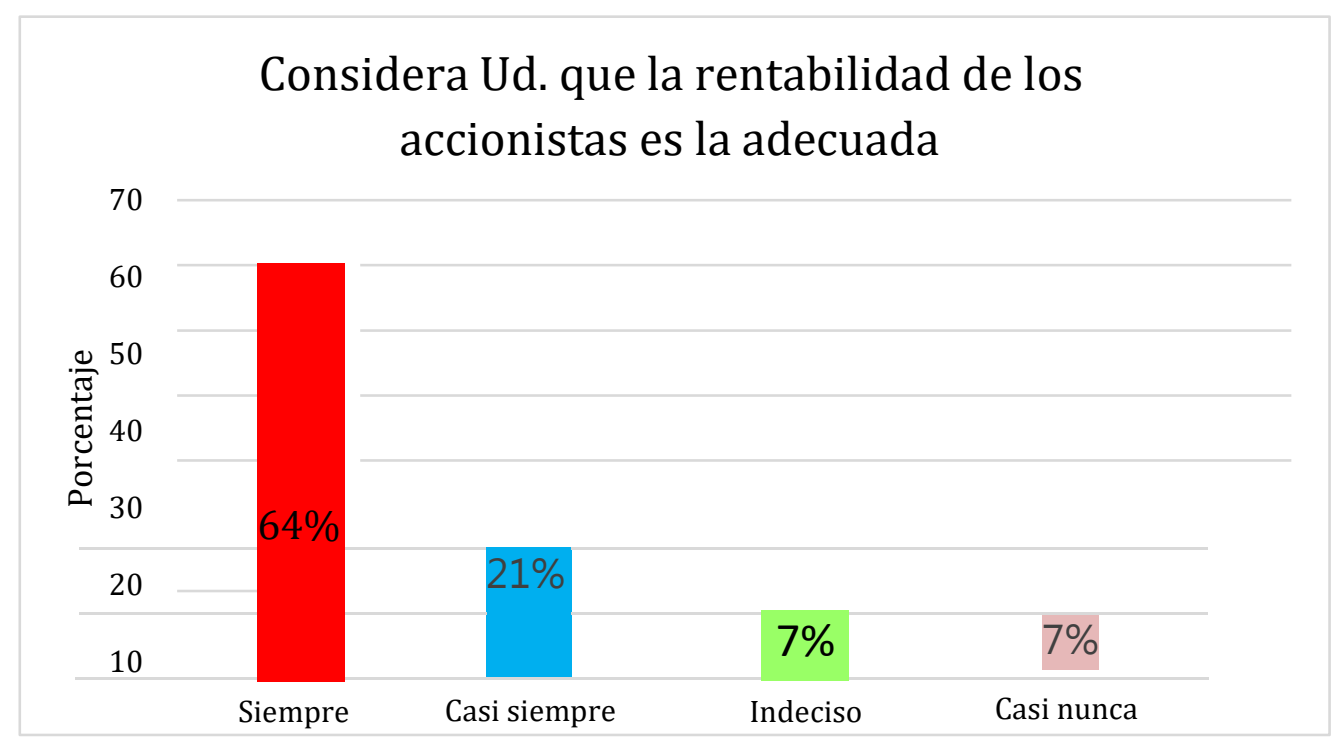

Gráfico 4. La empresa genera una rentabilidad positiva sobre las ventas

En el gráfico 4 se puede apreciar que el $64 \%$ de los colaboradores consideran que la rentabilidad de los accionistas siempre es la adecuada, el $21 \%$ considera que casi siempre y el $7 \%$ está indeciso. 


\section{Prueba de hipótesis general}

- H0: No existe relación significativa entre el control interno de inventarios y la rentabilidad de la empresa HECALIROJA JIA SAC Tarapoto.

- H1: Sí existe relación significativa entre el control interno de inventarios y la rentabilidad de la empresa HECALIROJA JIA SAC Tarapoto.
Regla de decisión:

- Si el p valor > 0,05 se acepta la Hipótesis Nula.

- Si el p valor < 0,05 se rechaza la Hipótesis Nula, por lo tanto se acepta la Hipótesis Alterna.

Tabla 3. Relación entre el control interno de inventarios y la rentabilidad de la empresa HECALIROJA JIA SAC Tarapoto.

\begin{tabular}{llll}
\hline Rentabilidad de la empresa & & & \\
\hline & Tau b & $\mathrm{p}$ & $\mathrm{n}$ \\
Control interno de inventarios &, $780^{* *}$ & valor & 14
\end{tabular}

**. La correlación es significativa en el nivel 0,01 (bilateral)

Fuente: Hidalgo, Bustos (2019)

La Tabla 3 muestra que, la relación entre el control interno de inventarios y la rentabilidad de la empresa obtuvieron un coeficiente Tau b de Kendall del 0,780** que indica una correlación positiva alta, y con un $p$ valor igual a $0,000(p<0,05)$. Por lo tanto, se rechaza la hipótesis nula $\mathrm{HO}$ y se acepta la hipótesis alterna $\mathrm{H} 1$, es decir, si existe relación significativa entre el control interno de inventarios y la rentabilidad de la empresa. Indicando que a mayor control interno de inventarios mayor será la rentabilidad de la empresa.

Tabla 4. Prueba de hipótesis específica. Relación entre las actividades de control y la rentabilidad de la empresa HECALIROJA JIA SAC Tarapoto.

\begin{tabular}{llll}
\hline Rentabilidad de la empresa & & \\
\hline & Tau b & $\mathrm{p}$ & $\mathrm{n}$ \\
Actividades de control &, $590^{* *}$ & valor & 14 \\
\hline
\end{tabular}

**. La correlación es significativa en el nivel 0,01 (bilateral)

Fuente: Hidalgo, Bustos (2019) 
La Tabla 4 muestra que, la relación entre las actividades de control y rentabilidad de la empresa obtuvieron un coeficiente Tau b de Kendall de 0,590** el cual indica una correlación positiva media, y con un $p$ valor igual a 0,000 $(p<0,05)$. Por lo tanto, se rechaza la hipótesis nula $\mathrm{HO}$ y se acepta la hipótesis alterna $H 1$, es decir, si existe relación significativa entre la dimensión actividades de control y rentabilidad de la empresa. Indicando que a mayores actividades de control de inventarios mayor será la rentabilidad de la empresa.

Tabla 5. Prueba de hipótesis específica. Relación entre la supervisión y monitoreo con la rentabilidad de la empresa HECALIROJA JIA SAC Tarapoto.

\section{Rentabilidad de la empresa}

\begin{tabular}{llll} 
& Rho & p & $\mathrm{n}$ \\
Supervisión y monitoreo &, $710^{* *}$ & valor &, 001 \\
\hline
\end{tabular}

**. La correlación es significativa en el nivel 0,01 (bilateral)

Fuente: Hidalgo, Bustos (2019)

La Tabla 5 muestra que, la relación entre la supervisión y monitoreo con la rentabilidad de la empresa obtuvieron un coeficiente Tau b de Kendall de 0,710** que indica una correlación positiva alta, y con un p valor igual a 0,001 $(p<0,05)$. Por lo tanto, se rechaza la hipótesis nula $\mathrm{HO}$ y se acepta la hipótesis alterna $\mathrm{H} 1$, es decir, si existe relación significativa entre la supervisión y monitoreo con la rentabilidad de la empresa HECALROJA JIA SAC Tarapoto. Indicando que, a mayor supervisión y monitoreo, mayor será la rentabilidad de la empresa.

\section{CONCLUSIONES}

De acuerdo a los resultados obtenidos en la presente investigación titulada "Control Interno de Inventarios y su Relación en la Rentabilidad de la Empresa HECALIRO JIA
SAC, Tarapoto", se llega a las siguientes conclusiones según los objetivos planteados:

En cuanto al objetivo general, llegamos a la conclusión que el control interno de inventarios se relaciona significativamente con la rentabilidad de la empresa HECALIRO JIA SAC ya que se obtuvo como resultado Tau b de Kendall $(\mathrm{t}=\mathbf{0} .780 * * ; \boldsymbol{p}=\mathbf{0}$. 000) el cual indica que a mayor control de inventarios mayor será la rentabilidad de la empresa., Tarapoto.

Respecto al primer objetivo específico, se determinó que existe relación significativamente entre las actividades de control y la rentabilidad de la empresa HECALIRO JIA SAC, indicando que a mayores actividades de control de inventarios que realicen mayor será la rentabilidad de la empresa. 
Con relación al segundo objetivo específico, se llegó a la conclusión que la dimensión supervisión y monitoreo tiene una relación positiva alta con la rentabilidad de la empresa HECALRO JIA SAC. El cual hace referencia que, a mayor supervisión $y$ monitoreo del control interno mayor será la rentabilidad de la empresa.

\section{REFERENCIAS}

Amat, J. (2015). Control 2.0. Una perspectiva del control de gestión menos financieray más cualitativa. Medellin: PLANETA

Área de Pymes (2015) Ratios de Rentabilidad recuperado

de:

http://www.areadepymes.com/?tit=ratios -de-rentabilidad-ratios-del-balancey-dela-cuenta-de-

resultados\&name $=$ Manuales $\&$ fid $=$ ej0bca $\mathrm{C}$

Boal N. (2015) Ratios de Rentabilidad, recuperado de: http://www.expansion.com/diccionarioeconomico/ratios-de-rentabilidad.html Cárdenas, M. (14 de Marzo de 2011). Slideshare. Recuperado el 2013, de http://www.slideshare.net/mcardenasmen dez/analisis- estructural-deestadosfinancieros

Chávez, J. (2016). Moderna Gestión de la Cadena de Suministros. Santiago: EDITORES

El Committee of Sponsoring Organizations of the Treadway Commission -COSO (2013) Control Interno - Marco Integrado Resumen Ejecutivo, Traducción al español, recuperado de: http://doc.contraloria.gob.pe/ControlInter no/Normativa_Asociada/coso_2013resumen-ejecutivo.pdf
Flores, M. (2018). Gestión de Inventarios y Rentabilidad. CEUPE, 30

Gerencie. (12 de junio de 2010). Recuperado el 30 de 11 de 2013, de http://www.gerencie.com/analisis-deestados-financieros.html

Gitman, L. (2007). Principios de Administración Financiera. México, D.F: Pearson. Gómez, G. (Agosto de 2001). GestioPolis. Recuperado el 2013, de http://www.gestiopolis.com/canales/finan ciera/articulos/no\%2016/analisisfinancier o.htm

Hernández Fernández Sampieri, B. (2014). Nueva Metodología de la Investigación. México: Planeta. Hemeryth y Sánchez (2013), en su tesis titulada "Implementación de un Sistema de control Interno operativo en los almacenes, para mejorar la gestión de los inventarios de la constructora A\&A S.A.C. de la

Ciudad de Trujillo - 2013" para optar por el grado de Contador Público y Licenciado en Administración, de la Universidad Privada Antenor Orrego de Trujillo - Perú

Mercale P. (2012) Estudio de la Rentabilidad de la Empresa recuperado de http://www.contabilidadempresa.com/2012/04/estudio-de-larentabilidadeconomica-y.html

Morales L. \& Torres M. (2015) en su tesis denominada: Diseño de un Sistema de Control Interno de inventario basado en el modelo Coso 1 para la empresa Service Lunch en la ciudad de Guayaquil, recuperado de: http://dspace.ups.edu.ec/bitstream/12345 6789/10008/1/UPS-GT001162.pd

Zoria, J. (2018). Gestión, una herramienta para el logro de objetivos. Mundo de negocios, 24 